\title{
Statistics versus dynamics: two methods for calculating the effective charge of colloidal particles
}

\author{
Alexandre Diehl ${ }^{1}$ and Yan Levin ${ }^{2}$ \\ ${ }^{1}$ Departamento de Física, Universidade Federal do Ceará, Caixa Postal 6030, CEP 60455-760, \\ Fortaleza, CE, Brazil \\ ${ }^{2}$ Instituto de Física, Universidade Federal do Rio Grande do Sul, Caixa Postal 15051, \\ CEP 91501-970, Porto Alegre, RS, Brazil \\ E-mail: levin@if.ufrgs.br
}

Received 28 September 2005

Published 28 October 2005

Online at stacks.iop.org/JPhysCM/17/S3309

\begin{abstract}
Two methods for calculating the effective charge of colloidal particles inside a salt free suspension are presented and analysed using Monte Carlo simulations. The first method is based on the Alexander prescription, in which the exact electrostatic potential is matched asymptomatically to the solution of the linearized Poisson-Boltzmann equation. The second method relies on a dynamical criterion, which defines the condensed counterions through a bound on their total energy. Although the two methods appear to be quite different, they lead to identical values of the effective colloidal charge, even for suspensions with multivalent counterions for which the Poisson-Boltzmann equation fails.
\end{abstract}

\section{Introduction}

The chemical (bare) charge of colloidal particles is produced by the dissociation of the colloidal surface groups synthesized to stabilize the suspension against flocculation. It is often found, however, that even if the surface groups are strong electrolytes and dissociated completely in an aqueous solution the counterions still remain in the vicinity of the colloidal surface [1-3]. The extent of the counterion-colloid association (condensation) depends largely on the colloidal surface charge, the valence of the counterions, and the presence of any other electrolyte inside the suspension. The colloid-counterion complex behaves as a new entity, the effective charge of which is determined by the number of condensed counterions [4].

The effective, renormalized, charge of the colloidal particle is of fundamental theoretical and experimental importance. It determines the electrophoretic mobility of colloids in an applied electric field as well as the strength of the interparticle interactions inside the suspension [5-20]. Notwithstanding its importance, our ability to explicitly calculate the renormalized charge is still quite limited. For suspensions containing only monovalent counterions and small concentration of 1:1 salt, the effective charge can be obtained by 
matching the solution of the full non-linear Poisson-Boltzmann (PB) equation to the asymptotic solution of the linearized $\mathrm{PB}$ equation with a renormalized charge [3, 4, 20-22]. This procedure is called the Alexander prescription. It is, however, well known that the PB equation fails when the correlations between the ions of electrolyte become important. This is particularly the case for suspensions containing multivalent counterions, or significant concentration of coions. Thus, the applicability of the PB equation is restricted to dilute suspensions containing monovalent counterions. To try to overcome this difficulty, we have recently proposed a different method for calculating the effective charge of salt free suspensions [23]. The new approach is based on a dynamical criterion which defines when a counterion is to be considered as associated with a colloid. The dynamical criterion is particularly simple to use within the framework of molecular dynamics or Monte Carlo simulations. We found that for suspensions containing monovalent counterions the effective charge obtained using the dynamical criterion is identical to the one found within the PB theory with the Alexander prescription. For multivalent counterions the $\mathrm{PB}$ equation fails and the effective charge derived on its basis shows an incorrect behaviour - the effective colloidal charge is found to saturate, while the dynamical criterion finds a maximum of the effective charge as a function of the bare charge [23, 24]. In this paper we will demonstrate that if the Alexander prescription is used directly within the Monte Carlo simulation and the exact electrostatic potential is asymptotically matched with the linearized solutions of the PB equation, the two methods lead, once again, to identical values of the renormalized charge.

\section{Poisson-Boltzmann cell model and the effective colloidal charge}

To study the effective charge of colloidal particles we use a cell model. A colloidal particle of radius $a$, and charge $-Z q$ is placed at the centre of a spherical Wigner-Seitz (WS), the radius $R$ of which is determined by the concentration of colloids inside the suspension. The cell also contains $Z / \alpha$ counterions of valence $\alpha$ and charge $+\alpha q$. The solvent is treated as a structureless medium of relative (in CGS units) dielectric constant $\epsilon$.

\subsection{Poisson-Boltzmann cell model}

The electrostatic potential inside the cell satisfies the Poisson equation. The local density of counterions at distance $r$ from the centre of the colloid is given by the Boltzmann distribution with exponent proportional to the potential of mean force between the colloid and a counterion, $w(r)$. The simplest approximation is to replace $w(r)$ by the electrostatic potential, $w(r) \approx \phi(r)$. This leads to a well known Poisson-Boltzmann (PB) equation

$$
\nabla^{2} \phi=-\frac{4 \pi}{\epsilon}[-Z q+\alpha q \rho(r)]
$$

where

$$
\rho(r)=\frac{Z \exp [-\beta \alpha q \phi(r)]}{4 \pi \alpha \int_{a}^{R} \mathrm{~d} r r^{2} \exp [-\beta \alpha q \phi(r)]} .
$$

It is important to stress that equation (2) is an approximation which is expected to work only when the correlations between the counterions are small.

To numerically solve the $\mathrm{PB}$ equation, it is convenient to rewrite it in terms of the electric field $\mathbf{E}(\mathbf{r})=-\nabla \phi(\mathbf{r})$. Substituting this expression into equation (1) and integrating, we find that the modulus of the electric field $E(r)$ satisfies an integral equation

$$
\mathcal{E}(x)=-\frac{1}{x^{2}}[Z-\alpha \gamma(x)]
$$


where $x=r / \lambda_{\mathrm{B}}, \mathcal{E}(x)=\beta q \lambda_{\mathrm{B}} E(r)$ and $\lambda_{\mathrm{B}}=\beta q^{2} / \epsilon$ is the Bjerrum length, and

$$
\gamma(x)=(Z / \alpha) \frac{\int_{\hat{a}}^{x} \mathrm{~d} x^{\prime} x^{\prime 2} \exp \left[-\alpha \int_{x^{\prime}}^{\hat{R}} \mathrm{~d} x^{\prime \prime} \mathcal{E}\left(x^{\prime \prime}\right)\right]}{\int_{\hat{a}}^{\hat{R}} \mathrm{~d} x^{\prime} x^{\prime 2} \exp \left[-\alpha \int_{x^{\prime}}^{\hat{R}} \mathrm{~d} x^{\prime \prime} \mathcal{E}\left(x^{\prime \prime}\right)\right]},
$$

with $\hat{a}=a / \lambda_{\mathrm{B}}$ and $\hat{R}=R / \lambda_{\mathrm{B}}$. We have taken the zero of the electrostatic potential to be at the cell boundary, $\phi(R)=0$. The electric field inside the cell is determined by a numerical iteration of equation (3) starting with an arbitrary function satisfying the boundary conditions $E(R)=0$ and $E(a)=-Z q / \epsilon a^{2}$, until the convergence is achieved.

\subsection{The effective charge within the Alexander prescription}

Alexander et al [3] proposed that the effective colloidal charge can be obtained by asymptotically matching the solution of the full non-linear PB equation to the solution of the linearized PB equation with an effective charge.

The asymptotic solution of the PB equation is obtained by expanding the counterion density to first order in electrostatic potential

$$
\rho(r)=\rho_{0}[1-\beta \alpha q \phi(r)],
$$

where

$$
\rho_{0}=\frac{Z}{4 \pi \alpha \int_{a}^{R} \mathrm{~d} r r^{2} \exp [-\beta \alpha q \phi(r)]} .
$$

Note that since $\phi(R)=0, \rho_{0}=\rho(R)$. The Poisson equation for $r>a$ reduces to

$$
\nabla^{2} \phi=G+\kappa^{2} \phi(r),
$$

where $G=-4 \pi \alpha q \rho_{0} / \epsilon$ and $\kappa^{2}=4 \pi \beta \alpha^{2} q^{2} \rho_{0} / \epsilon$ is the inverse screening length. Equation (7) can be solved to yield

$$
\phi(r)=-\frac{G}{\kappa^{2}}+A \frac{\mathrm{e}^{-\kappa r}}{r}+B \frac{\mathrm{e}^{+\kappa r}}{r},
$$

where the constants $A$ and $B$ are obtained from the boundary conditions, $\phi(R)=0$ and $\phi^{\prime}(R)=0$. Thus, if the value of $\rho_{0}=\rho(R)$ is known, then the exact asymptotic solution of the PB equation will be known as well. The difficulty is that in order to find $\rho_{0}$ one needs to have the complete solution of the non-linear PB equation. The Alexander prescription is then to numerically solve the non-linear PB equation to obtain $\rho(R)$. One then asks: if the linear solution is extended all the way to the colloidal surface, what colloidal charge does it correspond to? This is, precisely, the effective charge which is perceived by an observer far from the colloidal surface. Using the Gauss law and the solution of the linearized equation (8), the effective colloidal charge $Q_{\text {eff }}=-Z_{\text {eff }} q$ satisfies

$$
\frac{Q_{\text {eff }}}{\epsilon a^{2}}=-\left.\phi^{\prime}(r)\right|_{r=a} .
$$

Substituting the solution of the linearized PB equation, with coefficients $A$ and $B$ determined by the boundary conditions, we finally arrive at the expression for the effective charge

$$
Z_{\text {eff }}=\frac{\hat{G}}{2 \hat{\kappa}^{3}}\left[(\hat{\kappa} \hat{R}+1)(\hat{\kappa} \hat{a}-1) \mathrm{e}^{-\hat{\kappa}(\hat{R}-\hat{a})}-(\hat{\kappa} \hat{R}-1)(\hat{\kappa} \hat{a}+1) \mathrm{e}^{+\hat{\kappa}(\hat{R}-\hat{a})}\right],
$$

where $\hat{G}=\lambda_{\mathrm{B}}^{3} \epsilon G / q, \hat{\rho}(\hat{R})=4 \pi \lambda_{\mathrm{B}}^{3} \rho(\hat{R})$, and $\hat{\kappa}=\kappa \lambda_{\mathrm{B}}=\alpha[\hat{\rho}(\hat{R})]^{1 / 2}$ [25]. Thus, if $\rho(R)$ is known, $Z_{\text {eff }}$ can be determined by a direct application of the formula (10). At this point it is important to make the following observation. The non-linear PB equation 
fails when the correlations between the counterions become important. This is precisely the case when aqueous suspensions contain multivalent counterions. Thus, we might expect that equation (10), based on the PB theory, will also fail when $\alpha>1$. This, however, is not necessarily the case. While in the vicinity of a colloidal surface the counterion correlations are strong and the PB equation is not valid, close to the cell boundary the average separation between the counterions is quite large (unless the suspension is very concentrated) and the correlations between the counterions play no significant role. Under these conditions, the PB equation will remain valid near the cell boundary. Furthermore, for $r \approx R$ the electrostatic potential is weak and the $\mathrm{PB}$ equation can again be linearized leading to the general solution (8) and the effective colloidal charge (10). However, in this case $\rho(R)$ can no longer be calculated from the solution of the non-linear PB, but must be obtained by some other means, such as direct Monte Carlo simulation of the cell model. This is the route which will be taken in the present work.

\section{Dynamical effective charge and simulation method}

Recently we proposed a new method for calculating the effective colloidal charge of salt free suspensions [23]. A counterion is considered condensed if and only if its total energy is sufficiently negative,

$$
U \leqslant-\chi K
$$

where $K=p^{2} / 2 m$ is the counterion kinetic energy and $U$ is its electrostatic energy. For suspensions containing monovalent counterions, the condensation criterion (11) with $\chi=4 / 3$ was found to lead to effective colloidal charges identical to the ones obtained using the Alexander prescription and the PB theory. For multivalent counterions, however, the PB theory and equation (11) resulted in very different effective charges [23].

Implementation of the criterion (11) within the canonical Monte Carlo (MC) simulation [26] is quite straightforward. The initial configuration is equilibrated during $(1-10) \times 10^{6} \mathrm{MC}$ steps, where we attempted to move a counterion to a new position, using a $1 / r$ biased sampling to speed up the simulation. Production runs amount to $(10-200) \times 10^{6}$ MC steps, producing 1000 uncorrelated configurations for observable averages. For each one of these uncorrelated configurations, we assigned a momentum vector $\mathbf{p}=\left(p_{x}, p_{y}, p_{z}\right)$ to each counterion, with the component $p_{i}$ taken from the normalized Maxwell-Boltzmann distribution. The number of condensed counterions $n^{\star}$ for each equilibrium configuration is then determined using the criterion (11). The effective colloidal charge is defined as $Z_{\text {eff }}=Z-\alpha \bar{n}^{\star}$, where $\bar{n}^{\star}$ is the average (over various equilibrium configurations) number of condensed counterions.

In this paper we will compare the effective charges obtained using the criterion (11) with the ones found from equation (10) with $\rho(R)$ calculated using MC simulation.

\section{Results and discussion}

It is convenient to define the total number of counterions within a radial distance $r$ from the colloidal centre as

$$
P(r)=4 \pi \int_{a}^{r} \mathrm{~d} r^{\prime} r^{\prime 2} \rho\left(r^{\prime}\right)
$$

Electroneutrality inside the cell requires that $P(R)=Z / \alpha$. The counterion density at $r$ is then

$$
\rho(r)=\frac{1}{4 \pi r^{2}} \frac{\mathrm{d} P}{\mathrm{~d} r} .
$$




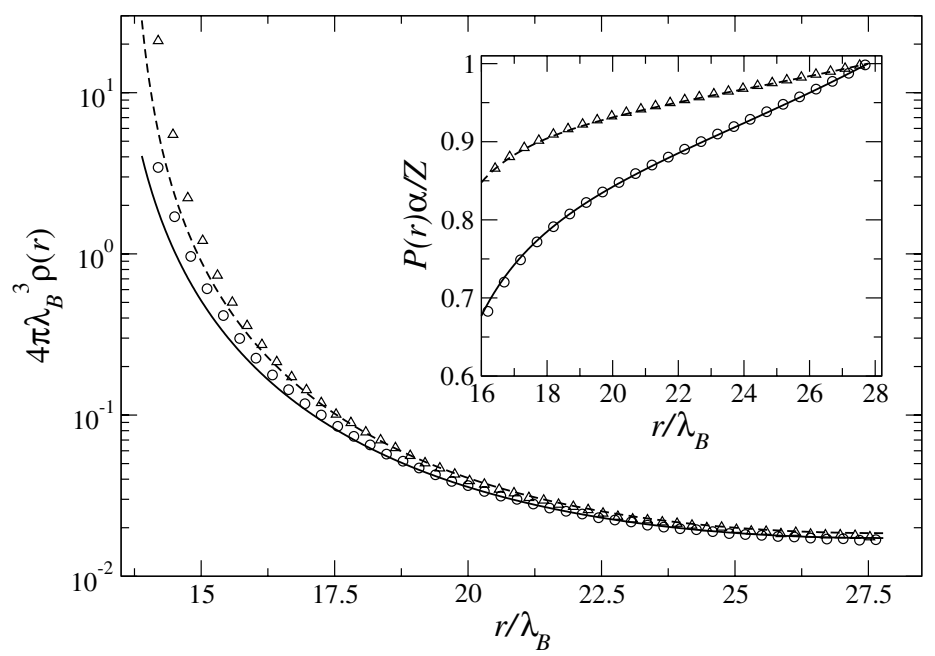

Figure 1. Monovalent counterion density profiles for colloidal particles of radius $a=100 \AA$ and charges $Z=600$ (open circles) and $Z=1500$ (open triangles), inside a WS cell of radius $R=200 \AA$. The symbols denote the results of the MC simulations, while the solid $(Z=600)$ and the dashed $(Z=1500)$ lines are obtained from the numerical solution of the Poisson-Boltzmann equation (3) and equation (2). The inset shows the integrated number of counterions close to the cell boundary.

The integrated number of counterions $P(r)$ can be obtained from the MC simulation using the following procedure. The WS cell is divided into concentric equidistant shells (bins) and the average number of counterions in each bin is calculated. The integrated charge $P(r)$ is then obtained by summing up all the bins up to a given $r$. The density profile, $\rho(r)$, is found using equation (13) by first performing a polynomial interpolation of $P(r)$, followed by a differentiation.

In figure 1 we compare the simulation results with the numerical solution of the nonlinear PB equation (1). The radius of the colloid is $a=100 \AA$ and of the WS cell $R=200 \AA$, corresponding to a volume fraction $\phi=(a / R)^{3}=0.125$, the solvent is water at room temperature, $\lambda_{\mathrm{B}}=7.2 \AA$. The colloidal charges are $Z=600$ and 1500 , while the counterions are monovalent hard spheres of radius $a_{\mathrm{c}}=2 \AA$. The overall agreement between the PB equation and the MC simulations is quite satisfactory. The PB equation fails close to the colloidal surface, since in this region the density of the counterions is large and the correlations appear to be significant even for monovalent counterions. When the valence of the counterions is increased, the deviation from the PB solution becomes significant over the whole span of the cavity, as is shown in figure 2 for trivalent counterions.

We next look at the effective charge calculated using the three different methods: (A) Alexander prescription, equation (10), and the PB theory; (B) equation (10), but using $\rho(R)$ obtained directly from the Monte Carlo simulation; and (C) condensation criterion, equation (11), with $\chi=4 / 3$.

In figure 3 we show the comparison between the three effective charges calculated for colloidal particles of radius $a=100 \AA$ inside a WS cell of $R=200 \AA$. It is evident that while for monovalent counterions the three methods lead to very similar values of the effective charge, in the case of the multivalent counterions method (A) fails completely [23, 24]. On the other hand, method (B), based on the asymptotic solution of the PB equation, produces 


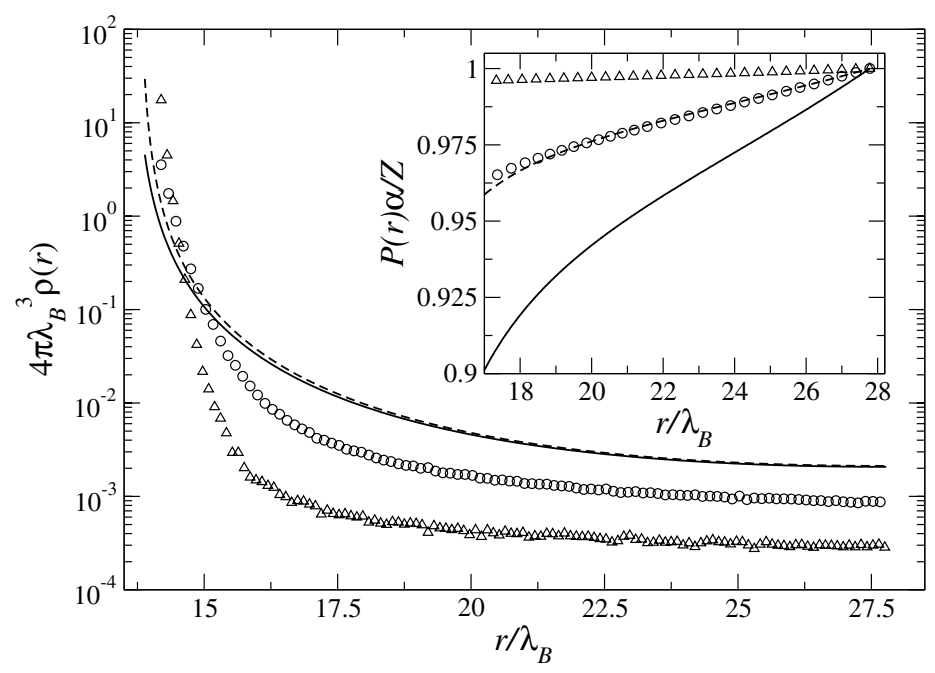

Figure 2. Trivalent counterion density profiles for a colloidal particle of radius $a=100 \AA$ inside a WS cell of radius $R=200 \AA$. The symbols and lines are the same as in figure 1 . Note that close to the cell boundary the PB density profile is independent of the bare charge $Z$. This leads to a well known saturation of the $Z_{\text {eff }}$ within the PB theory. This behaviour, however, is incorrect for multivalent counterions, as can be seen from the MC data. For multivalent counterions $\rho(r)$ does depend on $Z$ even at the cell boundary. The inset shows the integrated number of counterions close to the cell boundary.

effective charges in perfect agreement with the condensation criterion—even for multivalent counterions.

The correlations between the counterions which are responsible for the failure of the PB equation are the strongest in the vicinity of the colloidal surface. Thus if, for the same $Z$, colloidal radius is increased - decreasing the colloidal surface change density-the average distance between the condensed counterions will diminish, and so will the role of the electrostatic correlations. This is precisely what is found in figure 4. For colloids of $a=720 \AA$, all three methods give very similar results for the effective charge, even in the case of multivalent counterions. The relevance of electrostatic correlations for counterions of various valences can be quantified by a plasma parameter $[4,23]$

$$
\Gamma=\frac{\alpha^{\frac{3}{2}} \lambda_{\mathrm{B}} \sqrt{Z}}{4 a} .
$$

If $\Gamma<1$ the electrostatic correlations are not important and the PB theory should be applicable (unless the counterions are very large [27]). For $\Gamma>1$ the correlations are important and the PB equation fails $[28,29]$. The effective charge in the latter case must be obtained using either method (B) or (C).

\section{Conclusions}

We have studied three different methods for calculating the effective charge of colloidal particles in a salt free suspension. It was found that both the Alexander prescription and the condensation criterion (11) lead to identical values of the effective charge. This remains true even for highly charged suspensions with multivalent ions for which the Poisson-Boltzmann 


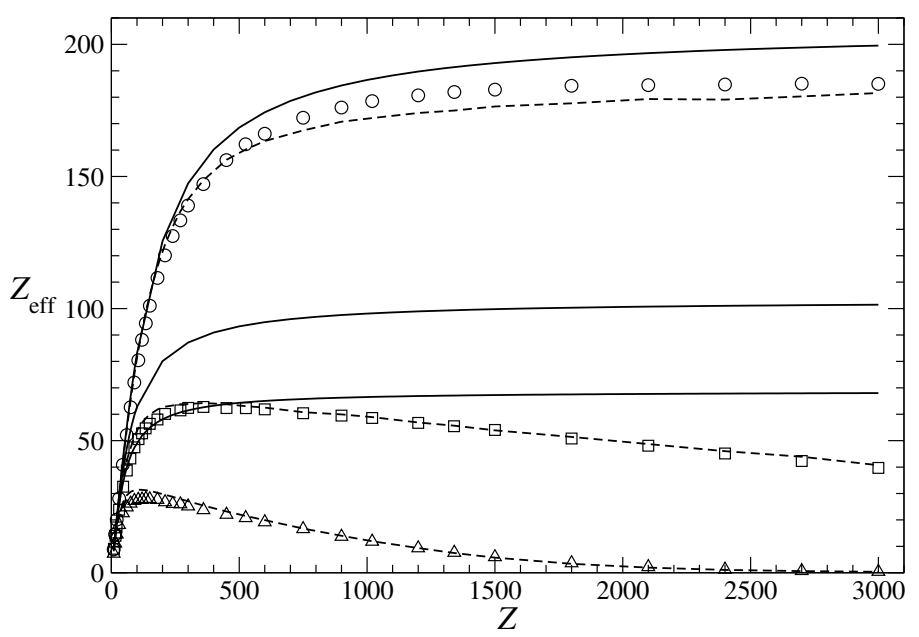

Figure 3. The effective charges of colloidal particles of radius $a=100 \AA$ inside a WS cell of $R=200 \AA$, as a function of the bare charge $Z$. The symbols are the effective charges obtained from the MC simulations using the condensation criterion (11). The counterion valence is: $\alpha=1$ (open circles), $\alpha=2$ (open squares), and $\alpha=3$ (open triangles). The solid lines were calculated using the Alexander prescription, equation (10), and the non-linear PB equation (from top to bottom for $\alpha=1,2,3$ ). The dashed lines (from top to bottom) were calculated using the equation (10), and the density $\rho(R)$ obtained from the MC simulations. Statistical uncertainties are smaller than the symbol size. Note that for divalent counterions the plasma parameter, $\Gamma=1$ when $Z=380$, and for trivalent when $Z=115$. These values are consistent with the location of the maximum of $Z_{\text {eff }}$.

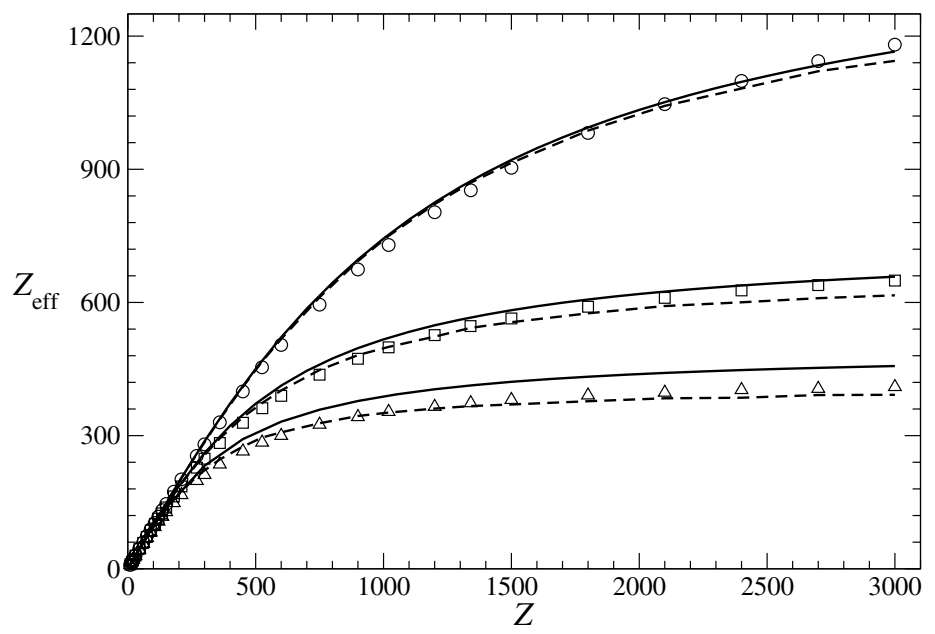

Figure 4. The effective charge of colloidal particles of radius $a=720 \AA$ inside a WS cell of $R=1440 \AA$. The symbols and lines are the same as in figure 3 .

equation-on which the Alexander prescription is based-is no longer valid. In this case, however, the density at the cell boundary which enters equation (10) must be taken directly from the Monte Carlo or molecular dynamics simulation. 
Unfortunately, at this point there does not appear to be a straightforward way of extending the formalism developed above to highly charged suspensions containing multivalent counterions as well as 1:1 electrolyte. Under these conditions, both the condensation criterion (11) and the formula for the effective charge (10) lose their validity. The Alexander prescription fails because the correlations between the counterions and coions remain important even far from the colloidal surface, and the PB equation ceases to be valid even asymptotically. Similarly, in the presence of coions, the condensation criterion (11) fails to distinguish if the counterion is 'condensed' because of its interaction with the colloid, or because of the presence of a nearby coion. A reliable method for obtaining the effective colloidal charge under these conditions remains to be found.

\section{Acknowledgments}

This work was supported in part by the Brazilian agencies CNPq, Capes and Fapergs.

\section{References}

[1] Manning G S 1969 J. Chem. Phys. 51924

[2] Manning G S 1978 Q. Rev. Biophys. II 2179

[3] Alexander S, Chaikin P M, Grant P, Morales G J, Pincus P and Hone D 1984 J. Chem. Phys. 805776

[4] Levin Y 2002 Rep. Prog. Phys. 651577

[5] Torrie G M and Valleau J P 1980 J. Chem. Phys. 735807

[6] Lozada-Cassou M, Saavedra-Barrera R and Henderson D 1982 J. Chem. Phys. 775150

[7] Belloni L 1986 J. Chem. Phys. 85519

[8] Belloni L 1998 Colloids Surf. A 140227

[9] Quesada-Perez M, Gonzalez-Tovar E, Martin-Molina A, Lozada-Cassou M and Hidalgo-Alvarez R 2003 Chem. Phys. Chem. 4235

[10] Martin-Molina A, Quesada-Perez M, Galisteo-Gonzalez F and Hidalgo-Alvarez R 2003 J. Phys.: Condens. Matter 15 S3475

[11] Garbow N, Eyers M, Palberg T and Okubo T 2004 J. Phys.: Condens. Matter 163835

[12] Grosberg A Y, Nguyen T T and Shklovskii B I 2002 Rev. Mod. Phys. 74329

[13] Besteman K, Zevenbergen M A G, Heering H A and Lemay S G 2004 Phys. Rev. Lett. 93170802

[14] Fernández-Nieves A, Fernández-Barbero A and de las Nieves F J 2000 Langmuir 164090

[15] Gelbart W M, Bruinsma R F, Pincus P A and Parsegian V A 2000 Phys. Today 5338

[16] Hansen J P and Löwen H 2000 Annu. Rev. Phys. Chem. 51209

[17] Tanaka M and Grosberg A Y 2001 J. Chem. Phys. 115567

[18] Tanaka M 2003 Phys. Rev. E 68061501

[19] Solis F J and de la Cruz M O 2001 Phys. Today 5471

[20] Trizac E and Levin Y 2004 Phys. Rev. E 69031403

[21] Trizac E, Bocquet L and Aubouy M 2002 Phys. Rev. Lett. 89248301

[22] Bocquet L, Trizac E and Aubouy M 2002 J. Chem. Phys. 1178138

[23] Diehl A and Levin Y 2004 J. Chem. Phys. 12112100

[24] Groot R D 1991 J. Chem. Phys. 959191

[25] Trizac E, Bocquet L, Aubouy M and von Grunberg H 2003 Langmuir 194027

[26] Allen M P and Tildesley D J 1987 Computer Simulations of Liquids (Oxford: Oxford University Press)

[27] Messina R, Gonzalez-Tovar E, Lozada-Cassou M and Holm C 2002 Europhys. Lett. 60383

[28] Moreira A G and Netz R R 2000 Europhys. Lett. 52705

[29] Naji A, Jungblut S, Moreira A G and Netz R R 2005 Physica A 352131 